\title{
XIX. On the laws of reflexion from crystallized surfaces
}

\section{J. MacCullagh}

To cite this article: J. MacCullagh (1836) XIX. On the laws of reflexion from crystallized surfaces, Philosophical Magazine Series 3, 8:45, 103-108, DOI: $\underline{10.1080 / 14786443608648815}$

To link to this article: http://dx.doi.org/10.1080/14786443608648815

曲 Published online: 01 Jun 2009.

Submit your article to this journal \lceil

Џ Article views: 1

Q View related articles $\sqsubset$ 
and whether there is any limit to the numbers of the orders of the combining atoms."

Under these circumstances, it would have been better not to construct any mineralogical formulæ, except the simple ones; and in future the composition of such minerals as involve the uncertain orders of atoms should be expressed simply by the proportion of each ingredient in 1000th parts, or by the nearest equivalent numbers of each, taking the element which occurs in the smallest quantity as an atomic unit. We should then express by our formulæ what we know, instead of contriving to represent an imaginary atomic constitution, which, if the atomic theory be true, is probably in all cases false.

January 7 th, 1836.

I am, dear Sir, yours truly,

H. J. Brooke.

XIX. On the Laws of Reflexion from crystallized Surfaces. By J. MacCullagh, Fellow of Trinity College, Dublin.

Dear Sir,

To Sir David Brewster.

T HAVE great pleasure in sending you an account of the 1 laws by which I conceive that the vibrations of light are regulated when a ray is reflected and refracted at the separating surface of two media; especially as the only guide which $I$ had, in my inquiry after these laws, was your paper on the action of crystallized surfaces upon light, published in the Philosophical Transactions for the year 1819. The observation which I found there, that the polarizing angle was the same for a given plane of incidence, "whether the obtuse angle of the rhomb [of Iceland spar] was nearest or furthest from the eye, or whether it was to the right or left hand of the observer," disappointed me at first, being contrary to what I had anticipated from principles analogous to those which had been employed by Fresnel in the problem of reflexion from ordinary media. I then sought other principles, and the observation is now a result of theory.

Assuming, as a basis for calculation, that Fresnel's law of double refraction is rigorously true, $I$ have been obliged to make an essential change in his physical ideas. Conceive an ellipsoid whose semiaxes are parallel to the three principal directions of the crystal, and equal respectively to its three principal indices of refraction, and let a central section of the ellipsoid be made by a plane parallel to the plane of a wave passing through the crystal. The section will be an ellipse, and the wave will be polarized by the crystal in a plane pa- 
rallel to either semiaxis of this ellipse, the index of refraction for the wave being equal to the other semiaxis. This is Fresnel's law of double refraction; and the theory which led him to it makes it necessary to admit that the vibrations of the wave are perpendicular to its plane of polarization; whereas, according to the views which I have adopted, the vibrations of the wave are parallel to its plane of polarization, and to one semiaxis of the elliptic section, while its index of refraction is equal to the other semiaxis. These views nearly agree with the theory of M. Cauchy, according to whom the vibrations of polarized light are parallel to its plane of polarization, but inclined at small angles to the plane of the wave in crystallized media, instead of being exactly parallel to the latter plane, as I have supposed them to be. Besides, the theory of M. Cauchy, founded on the six equations of pressure in a crystallized medium, implies the existence of a third ray of feeble intensity, and for the other two rays gives a law somewhat different from that of Fresnel. Being obliged, in order to account for your experiments, to abandon the physical ideas of Fresnel, and to approximate towards those of M. Cauchy, I was embarrassed by this third ray; and wishing to get rid of it, as well as of the slight deviations from the symmetrical law of Fresnel, I adopted the expedient of altering the equations of pressure, in such a way as to make them afford only two rays, and give a law of refraction exactly the same as Fresnel's. The equations which I found to answer this purpose are the following:

$$
\begin{aligned}
& \mathrm{A}=-2\left(c^{2} \frac{d \eta}{d y}+b^{2} \frac{d \zeta}{d z}\right) \mathrm{V}^{2} \rho, \\
& \mathrm{B}=-2\left(a^{2} \frac{d \zeta}{d z}+c^{2} \frac{d \xi}{d x}\right) \mathrm{V}^{2} \rho, \\
& \mathrm{C}=-2\left(b^{2} \frac{d \xi}{d x}+a^{2} \frac{d \eta}{d y}\right) \mathrm{V}^{2} \rho, \\
& \mathrm{D}=a^{2}\left(\frac{d \eta}{d z}+\frac{d \zeta}{d y}\right) \mathrm{V}^{2} \varrho, \\
& \mathbf{E}=b^{2}\left(\frac{d \xi}{d z}+\frac{d \zeta}{d x}\right) \mathrm{V}^{2} \rho, \\
& \mathrm{F}=c^{2}\left(\frac{d \xi}{d y}+\frac{d \eta}{d x}\right) \mathrm{V}^{2} \varrho .
\end{aligned}
$$

In these equations, the axes of coordinates are perpendicular to each other, and parallel to the three principal directions 
of the crystal ; $x, y, z$ are the coordinates of a vibrating molecule at the time $t ; \xi, \eta, \zeta$ are the components of the displacement of the same molecule at the same time; $a, b, c$ are the three principal indices of refraction out of the crystal into an ordinary medium in which the velocity of light is equal to $\mathrm{V}$; and $\rho$ is the density of the æther, which density I suppose to be the same in all media. The quantities A, F, E are the components, parallel to the axes of $x, y, z$ respectively, of the pressure upon a plane perpendicular to the axis of $x ; \mathrm{F}, \mathrm{B}, \mathrm{D}$ are the components of the pressure upon a plane perpendicular to the axis of $y$; and $\mathrm{E}, \mathrm{D}, \mathrm{C}$, the components of the pressure upon a plane perpendicular to the axis of $z$. The values of D, E, F are the same as those given by M. Cauchy; but the values of $\mathrm{A}, \mathrm{B}, \mathrm{C}$ are different from his, and much simpler. By introducing into the equations of M. Cauchy the condition that the vibrations shall be performed without any change of density, the resulting values of $\mathrm{A}, \mathrm{B}, \mathrm{C}$ might be shown to agree nearly with those given above. The six pressures, A, B, C, D, E, F, being known, it is easy to find the pressure upon a plane making any given angles with the axes of coordinates.

These things being premised, it is time to mention the laws, or rather hypotheses, which I have imagined for discovering the relations that exist, as to direction and magnitude, among the vibrations in each ray, when reflexion and refraction take place at the separating surface of two media, whether crystallized or not. In stating the two very simple laws that have occurred to me for this purpose, it will be convenient, when the first medium is an ordinary one, to suppose that the incident light is polarized. Then by the first law, the vibrations in one medium are equivalent to those in the other; that is to say, if the incident and reflected vibrations be compounded, like forces acting at a point, their resultant will be the same, both in length and direction, as the resultant of the refracted vibrations similarly compounded. - By the second law, the $l a$ teral pressure upon the separating surface is the same in both media; the lateral pressure being understood to mean the pressure in a direction perpendicular to the plane of incidence.

As it would engage us too long to follow these laws into detail, I shall merely state some of the results which I have obtained from them, for the case of a uniaxal crystal into which the light passes out of an ordinary medium.

Imagine the surface of the crystal to be horizontal, and call the point of incidence $I$. With the centre $I$ and any radius, conceive a sphere to be described, cutting in the point $Z$ a vertical line I $\mathrm{Z}$ drawn through the centre, and let a radius

Third Series. Vol. 8. No. 45. Feb. 1836. 
I P, parallel to the axis of the crystal, meet the surface of the sphere in $\mathrm{P}$. Let the great circle $\mathrm{ZO} \mathrm{O}$ be the plane of incidence, containing both the direction IO of the ordinary refracted ray produced backwards, and the direction $I \mathrm{E}$ of a normal to the extraordinary wave; and

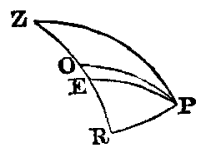
draw the great circles $\mathrm{PZ}, \mathrm{PO}, \mathrm{PE}$. The angle $Z$ will be the azimuth of the plane of incidence. Let $\mathrm{ZO}=\phi, Z E=\phi^{\prime}, \mathrm{PO}=\psi, \mathrm{PE}=\psi^{\prime}$, the angle $\mathrm{ZO} \mathrm{P}$ $=\theta$, and the angle $\mathrm{ZEP}=\theta^{\prime}$. Call the angle of incidence $i$, and suppose $b$ to be the reciprocal of the ordinary refractive index, and $a$ the reciprocal of the extraordinary.

Each of the refracted rays, in turn, may be made to disappear, by polarizing the incident ray in a certain direction assigned by theory. When the extraordinary ray disappears, the reflected ray is polarized in a plane inclined to the plane of incidence at an angle $\beta$ determined by the formula

$\tan \beta=\cos (i+\phi) \tan \theta+2\left(a^{2}-b^{2}\right) \sin \theta \sin \psi \cos \psi \frac{\sin ^{2} i}{\sin (i-\phi)}$

When the ordinary ray disappears, the plane of polarization of the reflected ray is inclined to the plane of incidence at an angle $\beta^{\prime}$ determined by the formula

$$
\begin{gathered}
-\tan \beta^{\prime}=\cos \left(i+\phi^{\prime}\right) \operatorname{cotan} \theta^{\prime}+\left(a^{2}-b^{2}\right) \frac{\cos 2 \theta^{\prime}}{\sin \theta^{\prime}} \\
\sin \psi^{\prime} \cos \psi^{\prime} \frac{\sin ^{2} i}{\sin \left(i-\dot{\phi}^{\prime}\right)^{\circ}}
\end{gathered}
$$

And when the angles $\beta, \beta^{\prime}$, become equal, the plane of polarization of the reflected ray becomes independent of the plane of polarization of the incident ray; and the angle of incidence $i$, at which this equality takes place, is the polarizing angle of the crystal. Hence we have the equation of condition

$$
\left.\begin{array}{c}
\cos (i+\phi) \tan \theta+2\left(a^{2}-b^{2}\right) \sin \theta \sin \psi \cos \psi \frac{\sin ^{2} i}{\sin (i-\phi)} \\
+\cos \left(i+\phi^{\prime}\right) \operatorname{cotan} \theta^{\prime}+\left(a^{2}-b^{2}\right) \frac{\cos 2 \theta^{\prime}}{\sin \theta^{\prime}} \sin \psi^{\prime} \cos \psi^{\prime} \sin ^{2} i \\
\sin \left(i-\phi^{\prime}\right)
\end{array}\right\}=0 .(4 .)
$$

to be fulfilled at the polarizing angle.

Since $i+\phi$, in this equation, is nearly equal to a right angle, put $i+\phi=\frac{\pi}{2}+\delta$, and $\delta$ will be a small quantity. Draw $\mathrm{P} R$ an arc of a great circle perpendicular to $\mathrm{ZOE}$, and let 
$\mathrm{Z} \mathrm{R}=p, \mathrm{P} \mathrm{R}=q$. Then we shall find from equation (4.), after various substitutions and reductions,

$$
\delta=\mathrm{K} \cos ^{2} q\left(\cos ^{2} \phi-\cos ^{2} p\right) ; \text { where } \mathrm{K}=\frac{\left(a^{2}-b^{2}\right)\left(1+b^{2}\right)}{2 b\left(1-b^{2}\right)} \text {. }
$$

In deducing this value of $\delta$, the approximations were made with a tacit reference to the case of reflexion in air from a common rhomb of Iceland spar. The coefficient $\mathrm{K}$, in this case, is equal to about nine degrees, and the resulting numerical values of the polarizing angles in various azimuths agree very well with your experiments. You will perceive that the value of $\delta$ is the same in supplementary azimuths, which explains the observation, cited in the beginning of my letter, relative to the equality of the polarizing angles at opposite sides of the perpendicular $I Z$ in a given plane of incidence.

When the point $\mathrm{R}$ falls upon $\mathrm{O}$, we have $\delta=0$, and $i+\phi$ equal to a right angle. Hence, when the cotangent of $\mathrm{Z} \mathrm{R}$ is equal to the ordinary index, the tangent of the polarizing angle is equal to the same index. This theorem, though deduced from an approximate equation, might be shown to be exact.

When the axis of the crystal lies in the plane of incidence, we may obtain an exact expression for the polarizing angle. The condition of polarization then becomes

$$
\cos \left(i+\phi^{\prime}\right)-\left(a^{2}-b^{2}\right) \sin \psi^{\prime} \cos \psi^{\prime} \frac{\sin ^{2} i}{\sin \left(i-\phi^{\prime}\right)}=0 ;
$$

from which, by the proper substitutions, we obtain the following expression :

$$
\sin ^{2} i=\frac{1-a^{2} \cos ^{2} \lambda-b^{2} \sin ^{2} \lambda}{1-a^{2} b^{2}} ;
$$

where $\lambda$ denotes the complement of $Z P$, or the inclination of the axis to the face of the crystal, and $i$ is the polarizing angle. This formula, in a shape somewhat different, was communicated, above a year ago, to Professor Lloyd, who has noticed it, in connexion with your paper, in his Report on Physical Optics. When $a$ and $b$ become equal, the formula gives your law of the tangent for ordinary media.

The foregoing results show that, when a ray is polarized by reflexion from a crystal, the plane of polarization deviates from the plane of incidence, except when the axis lies in the latter plane; and that the deviation may be made very great by placing the crystal in contact with a medium whose refractive power is nearly equal to that of the crystal itself; for when $i$ is nearly equal to $\phi$ or to $\phi^{\prime}$, the divisor sin $(i-\phi)$ or $\sin \left(i-\phi^{\prime}\right)$ is very small, and therefore $\tan \beta$ or $\mathrm{O} 2$ 
$\tan \beta^{\prime}$ is very great. But this remark is of no value whatever in explaining the very singular phænomena which you have observed in the extreme case just mentioned; nor can I imagine any reason why there should be a deviation, as there was in some of your experiments, when the axis lies in the plane of incidence, since everything is then alike on both sides of this plane. Indeed the whole of this subject, which occupies the latter part of your paper of 1819 , is very extraordinary and interesting; and I was glad to hear that you had resumed the investigation of it, and made many experiments which have not been published.

I wish you would publish them. They seem to be of great importance in the present state of optical science. I am, dear Sir, ever truly yours,

Trin. Coll. Dublin, Dec. 22, 1835. J. MacCuliagh.

XX. Some further Remarks on the Magnetic Forces. By R. W. Fox.*

I AM glad that Dr. Ritchie has noticed my remarks on the laws of the magnetic forces, because I hope that it will be the means of exciting more attention to the subject. I cannot, however, admit the justness of his conclusion, unless it can be shown that the results of my experiments are conformable to the law of the inverse of the squares of the distances throughout the rohole series of nine or ten removals of the magnet, calculating from any assumed points whatever in them. Dr. Ritchie has confined his calculations to only two or three distances.

The magnets which I employed were cylinders of three inches long and one tenth of an inch in diameter, and attracted each other with half the force of contact when separated about $\frac{1}{2} \frac{1}{0 \sigma} \sigma$ of an inch. From this minute distance to that of $\frac{1}{8}$ and even $\frac{1}{4}$ of an inch, the results were nearlyt in accordance with the law of the simple inverse ratio of the distance, calculating from the contiguous surfaces of the magnets; and when the same bars were made more strongly magnetic, their force, at half an inch, much more nearly approximated to the simple, than to the duplicate, inverse ratio of the distance.

* Communicated by the Author.

+ I have used this qualifying word, because at very minute distances the diminution of the force did not seem to be quite equal to the inverse ratio of the distance; whereas it rather exceeded it towards the end of the series. At the distance of $\frac{T}{4}$ of an inch, the force, in the case referred to, was about $\frac{1}{26 \sigma}$ of that of contact. 